\title{
Índices cuantitativos en el ámbito de la investigación en el Perú, periodo 2000-2003
}

\author{
Jullo César Olaya Guerrero \\ Universidad Nacional Mayor de San Marcos
}

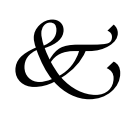

\section{Introducción}

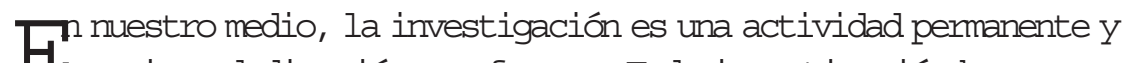
Irequiere dedicación y esfuerzo. Toda investigación busca ser difundida y valorada. El cONCYTEC (2001: 57) señala que "la innovación es la interacción entre las qportunidades del mercado y el conocimiento base de la empresa y sus capacidades" .

En el Perú existe el Instituto Nacional de Defensa de la Competencia y de la Propiedad Intelectual (INDECOPI) , creado en noviembre de 1992 para promover en la economía peruana una cultura de leal y honesta competencia y para proteger todas las formas de propiedad intelectual: desde los signos distintivos y los derechos de autor hasta las patentes y la biotecnología.

Para desarrollar esta investigación, hemos seleccionado información actualizada sobre innovación tecnológica y propiedad intelectual.

\section{Marco conceptual y teórico}

Las empresas buscan mejorar cada día. Ell resultado de esta búsqueda está íntimamente ligado a una realidad que concibe a "la empresa innovadora como aquella que ha implementado productos tecnológicamente nuevos o productos y procesos significativos mejorados", pues, "el proceso de innovación tecnológica implica la 
creación, desarrollo, uso y difusión de un nuevo producto, procesos o servicios nuevos y los cambios significativos de los mismos". (CONCYTEC 2001: 57).

Tenemos que aprender de los países desarrollados que invierten milllones de dólares en Investigación y Desarrollo (IyD). Es importante destacar el Manual de Oslo de OCDE que afirma que "las innovaciones tecnológicas hacen referencia tanto a los productos como a los procesos, así como a las modificaciones tecnológicas que se llevan a término en ellos". (Escorsa 1997: 22) .

La vigilancia tecnológica puede definirse como "la búsqueda, detección, análisis y commicación (a los directivos de la empresa) de informaciones orientadas a la toma de decisiones sobre amenazas y oportunidades extemas en el ámbito de la ciencia y tecnología". (Ashton y Klavams 1997) .

Existen dos herramientas decisivas para el acceso a la información: Bases de datos y la cienciometría. Podemos destacar que la cienciometría -bibliometría- se basa en el análisis y cómputo de determinados indicadores bibliométricos. Estos pueden ser de diverso tipo y van desde los autores de artículos hasta las citas que aparecen en la bibliografía de cada artículo pasando por las palabras contenidas en los títulos de los artículos o en los resúmenes.

INDECOPI en el año 2000, publicó un trabajo denominado Promoviendo un marco institucional para la valorización de la propiedad intelectual en el penú, que recoge una serie de propuestas concretas sobre los derechos de propiedad intelectual.

El Informe de Desarrollo Humano (PNUD 2001: 105-106), precisa que "actualmente los derechos de propiedad intelectual se están tornando más estrictos en todo el mundo. El número de patentes reivindicadas ha aumentado notablemente en los últimos 15 años, en los Estados Unidos de 77000 en 1985 a 169000 en 1999". (PNUD 2001: 105-106).

En el mes de abril del 2003, la Organización Mundial de la Propiedad Intelectual (OMPI) con el lema La propiedad intelectual es también asunto suyo, celebró el Día Mundial de la Propiedad Intelectual con el propósito de destacar el papel esencial de la propiedad intelectual en el éxito de las actividades empresariales y en la mejora de la vida cotidiana. El Dr. 
Kamil Idris, Director General de la Organización Mundial de la Propiedad Intelectual (OMPI), en su mensaje señala:

"En la actual economía de los conocimientos, los activos de propiedad intelectual son la divisa más fuerte. El éxito económico y los beneficios sociales y culturales que entraña dependen de la creación y gestión de la innovación, la información y las ideas. Encauzados por el sistema de propiedad intelectual, esos elementos impulsan de forma extraordinaria el desarrollo tecnológico y cultural y de ese modo generan el auge de nuevas industrias. La OMPI proclama que la propiedad intelectual es un valor universal que contribuye a la evolución del mundo y al progreso de las sociedades y es un elemento decisivo para el desarrollo económico, social y aultural a largo plazo. Ia creatividad es un rearso inagotable, propio de todas los pueblos y de importancia en todo tiempo y aultura". (OMPI 2003) .

\section{Resultados}

Los datos estadísticos fueron recopilados entre los meses de diciembre 2003 y enero del 2004, accediendo a bibliotecas especializadas de nuestro país.

\subsection{Innovación Tecnológica y Propiedad Intelectual}

La innovación tecnológica será medida a través de las patentes producidas, registros de propiedad intelectual, becas de estudios para postgrado en universidades nacionales e intemacionales, gastos en ciencia y tecnología, indicadores bibliométricas.

En el cuadro N. ${ }^{\circ} 1$, se muestran los datos de patentes de invención entre 1992 y 2002. 
Cuadro N. ${ }^{\circ} 1$

Patentes de invención solicitadas -otorgadas

en Perú, 1992-2002

\begin{tabular}{|c|c|c|c|c|}
\hline \multirow{2}{*}{ Años } & \multicolumn{2}{|c|}{$\begin{array}{c}\text { Solicitudes de } \\
\text { patentes de invención }\end{array}$} & \multicolumn{2}{c|}{$\begin{array}{c}\text { Patentes de invención } \\
\text { otorgadas }\end{array}$} \\
\cline { 2 - 5 } & Nacional & Extranj. & Nacional & Extranj. \\
\hline 1992 & 19 & 258 & 14 & 237 \\
\hline 1993 & 30 & 260 & 10 & 104 \\
\hline 1994 & 28 & 368 & 15 & 221 \\
\hline 1995 & 23 & 524 & 9 & 267 \\
\hline 1996 & 53 & 570 & 7 & 174 \\
\hline 1997 & 50 & 765 & 7 & 173 \\
\hline 1998 & 38 & 948 & 6 & 132 \\
\hline 1999 & 49 & 948 & 5 & 266 \\
\hline 2000 & 40 & 1040 & 9 & 299 \\
\hline 2001 & 36 & 944 & 14 & 523 \\
\hline 2002 & 31 & 824 & 22 & 528 \\
\hline
\end{tabular}

Fuente: Cuanto. Perú en números 2003. Lima: Cuanto, 2003; p. 317. Elaboración del autor, 2004.

Tomemos algunos años para el análisis. Del cuadro se desprende, por ejemplo, que las solicitudes de patentes fueron 855 en el año 2002, mientras que en el año 2000 se solicitaron 1080 patentes (nacionales y extranjeras). En el año 2002, las patentes otorgadas alcanzaron 550, mientras que el año 1999 fueron otorgadas 271 y en el año 2000, ascendieron a 308, respectivamente.

En lo referente a los registros de propiedad intelectual, se presenta el cuadro $\mathrm{N}^{\circ} 2$. 
Cuadro N. ${ }^{\circ} 2$

Registro de Propiedad Intelectual en Perú

2000-2003

\begin{tabular}{|c|c|c|}
\hline Años & $\begin{array}{c}\text { Registros } \\
\text { solicitados }\end{array}$ & $\begin{array}{c}\text { Registros } \\
\text { otorgados }\end{array}$ \\
\hline 2000 & 19054 & 14286 \\
\hline 2001 & 16719 & 15149 \\
\hline 2002 & 16672 & 13077 \\
\hline 2003 & 16695 & 13105 \\
\hline
\end{tabular}

Fuente: Cuanto. Perú en números 2001, p. 325.

Perú en números 2002, p. 320.

Perú en números 2003, p. 317.

Indecopi. http: //www. indecopi .gob.pe 2004 .

Elaboración del autor, 2004.

El cuadro N. ${ }^{\circ} 2$ registra datos muy importantes. En el año 2003, se solicitaron 16695 registros y se otorgaron 13 105. Mientras que, en el año 2000, las solicitudes llegaron a 19504 y fueron otorgados 14286 registros.

\subsection{Signos distintivos}

Entre 1993 y 1999, INDECOPI, a través de la Oficina de Signos Distintivos (OSD) , registró un total de 86106 signos distintivos, de los cuales 46\% se han realizado durante el período 1997-1998, lo que refleja una tendencia en la demanda de estos servicios. Del total registrado entre 1997 y 1999, el 68\% corresponde a marcas de productos, el $26 \%$ a marcas de servicios y el resto a lemas y nombres comerciales. (INDECOPI, 2000).

A continuación, se presenta el cuadro N. ${ }^{\circ} 3$. 
Cuadro N. ${ }^{\circ} 3$

Registro de signos distintivos

2000-2003

\begin{tabular}{|c|c|c|}
\hline Años & $\begin{array}{c}\text { Registros } \\
\text { solicitados }\end{array}$ & $\begin{array}{c}\text { Registros } \\
\text { otorgados }\end{array}$ \\
\hline 2000 & 16565 & 12724 \\
\hline 2001 & 14312 & 13258 \\
\hline 2002 & 14061 & 11452 \\
\hline 2003 & 14484 & 11424 \\
\hline
\end{tabular}

Fuente: Cuanto. Perú en Números 2001, p. 325.

Perú en Números 2002, p. 320.

Perú en Números 2003, p. 317.

Indecopi. http: //www. indecopi.gob.pe 2004.

Elaboración del autor, 2004.

El cuadro N. ${ }^{\circ} 3$, registra que en el año 2003 se solicitaron 14484 signos distintivos y se otorgaron 11 424. A comparación del año 2001, que se recibieron 14312 solicitudes y fueron otorgadas 13258 signos.

\subsection{Invenciones}

Durante el periodo 1997-1999, la Oficina de Invenciones otorgó 1184 registros. Estos se clasifican en concesión de patentes de invención (49\%), modelos de utilidad (7\%), registros de diseños industriales (40\%) y contratos de transferencia de tecnología (4\%). En lo que respecta a la composición por país de los solicitantes, el 35\% de los registros otorgadas correspande a titulares norteamericanos y el 26 \% a titulares peruanos. (INDECOPI 2000) .

A continuación, se presenta el cuadro N. ${ }^{\circ} 4$. 
Cuadro N. ${ }^{\circ} 4$

Registro de Invenciones

2000-2003

\begin{tabular}{|c|c|c|}
\hline Años & $\begin{array}{c}\text { Registros } \\
\text { solicitados }\end{array}$ & $\begin{array}{c}\text { Registros } \\
\text { otorgados }\end{array}$ \\
\hline 2000 & 1362 & 521 \\
\hline 2001 & 1234 & 816 \\
\hline 2002 & 1096 & 730 \\
\hline 2003 & 1170 & 735 \\
\hline
\end{tabular}

Fuente: Cuanto. Perú en números 2001, p. 325.

Perú en números 2002, p. 320.

Perú en números 2003, p. 317.

Indecopi. http: //www. indecopi.gob.pe 2004.

Elaboración del autor, 2004.

El año 2003 se solicitaron 1170 invenciones y fueron otorgadas 735. Mientras que en el año 2000, se recibieron 1362 solicitudes de invenciones y fueron otorgados 521 registros.

\subsection{Derechos de autor y derechos conexos}

Ell derecho de autor está íntimamente vinculado con la personalidad humana y contiene dos componentes: uno de naturaleza moral y otro patrimonial. Entre 1997 y 1999 la Oficina de Derechos de Autor del INDECOPI (ODA) otorgó 2919 registros, de los cuales el 70\% corresponden a obras literarias, 11\% a fonogramas, 9\% a software y 6\% a otras doras artísticas. (INDECOPI, 2000) .

En el cuadro N. ${ }^{\circ} 5$, se presenta los registros de derechos de autor entre los años 2000 y 2003. 
Cuadro N. ${ }^{\circ} 5$

Registro de Derechos de Autor

2000-2003

\begin{tabular}{|c|c|c|}
\hline Años & $\begin{array}{c}\text { Registros } \\
\text { solicitados }\end{array}$ & $\begin{array}{c}\text { Registros } \\
\text { otorgados }\end{array}$ \\
\hline 2000 & 1127 & 1041 \\
\hline 2001 & 1170 & 1075 \\
\hline 2002 & 975 & 895 \\
\hline 2003 & 1041 & 946 \\
\hline
\end{tabular}

Fuente: Cuanto. Perú en números 2001, p. 325.

Perú en números 2002, p. 320.

Perú en números 2003, p. 317.

Indecopi. http: //www. indecopi.gob.pe 2004.

Elaboración del autor, 2004.

Del cuadro N. ${ }^{\circ} 5$ se desprende que en el año 2003 se solicitaron 1041 registros de derechos de autor y fueron otorgados 946 registros. Mientras que en el año 2001, fueron registradas 1170 solicitudes y se otorgaron 1075.

\subsection{Becas de postgrado}

El Consejo Nacional de Ciencia y Tecnología, a través de la Oficina de Subvenciones, ofrece becas de estudios de postgrado en universidades nacionales e intemacionales, en forma amual.

A continuación se presenta el cuadro N. ${ }^{\circ} 6$. 
Cuadro N. ${ }^{\circ} 6$

Becas de estudios de postgrado

nacionales e internacionales

1997-2003

\begin{tabular}{|c|c|c|}
\hline Años & $\begin{array}{c}\text { Becas } \\
\text { nacionales }\end{array}$ & $\begin{array}{c}\text { Becas } \\
\text { internacionales }\end{array}$ \\
\hline 1997 & 135 & 31 \\
\hline 1998 & 93 & 33 \\
\hline 1999 & 140 & 42 \\
\hline 2000 & 120 & 54 \\
\hline 2001 & 160 & 143 \\
\hline 2002 & 147 & 74 \\
\hline 2003 & 100 & - \\
\hline
\end{tabular}

Fuente: Cuanto. Perú en Números 2002, p. 325.

Perú en Números 2003, p. 322.

Elaboración del Autor, 2004.

En el año 2003, se otorgaron 100 becas nacionales a personas interesadas en seguir estudios de postgrado y no se cuenta con datos disponibles de becas intemacionales. Sin embargo, en el año 2001, se ofrecieron 160 becas nacionales y 143 becas internacionales.

\subsection{Gastos en actividades de Ciencia y Tecnología}

En 1999, el gasto en actividades en ciencia y tecnología en el perú ascendió a 445 millones de dólares. En este gasto se incluyen los recursos destinados tanto a las universidades nacionales como a las instituciones de ciencia y tecnología. El gasto del Perú en ciencia y tecnología sólo representa el 0,89\% del PBI y la inversión en investigación y desarrollo solo asciende al 0,08 \% del PBI. (CONCYIFC, 2001) . En el año 2001, el gasto en ciencia y tecnología fue de solo 1,44\% en relación al PBI y en inversión de ciencia y tecnología ascendió al 0,11\%. El gasto de actividades en el 2001 fue de 779 millones de dólares. (RICYT, 2003) . 


\subsection{Indicadores bibliométricos}

Es importante remarcar que las publicaciones peruanas son registradas en grandes bases de datos, como Science Citation Index, Pascal o MEDLTNE. A continuación se presenta el cuadro № 7.

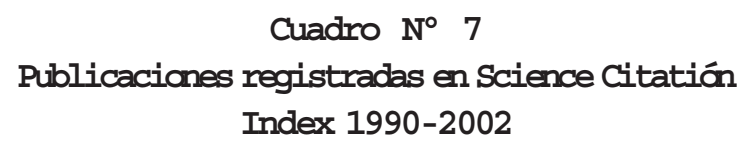

\begin{tabular}{|c|c|}
\hline Años & $\begin{array}{c}\text { Publicaciones } \\
\text { registradas en ISI }\end{array}$ \\
\hline 1990 & 163 \\
\hline 1991 & 173 \\
\hline 1992 & 143 \\
\hline 1993 & 169 \\
\hline 1994 & 130 \\
\hline 1995 & 177 \\
\hline 1996 & 180 \\
\hline 1997 & 173 \\
\hline 1998 & 183 \\
\hline 1999 & 186 \\
\hline 2000 & 228 \\
\hline 2002 & 244 \\
\hline
\end{tabular}

Fuente: Concytec, 2001, p. 135.

RICYT, http://www.ricyt.org 2003.

F. Cuevas, 2002.

El cuadro N. ${ }^{\circ} 7$ evidencia que en nuestro país se publica poco. Un dato significativo es el correspondiente al año 2002, donde la prooucción científica fue de 244 artículos del total de la producción mundial (1 145 654) . El 73\% de la producción fue realizada con la cooperación internacional y el 37\% de los trabajos publicados por los científicas peruanos fueron totalmente "hechos en casa". (Cuevas 2002) . 


\section{Conclusiones}

El estudio que hemos realizado evidencia que la innovación tecnológica en el Penú tiene matices significativos. Los investigadores no son conscientes de que deben registrar o patentar sus inventos en el INDECOPI.

La innovación tecnológica necesita ser apoyada por el Estado, las universidades deben ser las impulsadoras de la investigación científica y tecnológica.

La inversión en ciencia y tecnología en el Perú es mínima; no se publica mucho, no se difunde las investigaciones hechas en las universidades.

INDECOPI tiene programas de difusión de la propiedad intelectual que deben ser valorados por la población en su conjunto.

\section{Referencias bibliográficas}

BANCO CENIRAL DE RESERVA DEU PERÚ (2004) : Memoria del BCRP 2003. Banco Central de Reserva del Perú, Lima.

CONCYIEC (2001) : Indicadbres de ciencia, tecnología e innovación tecnológica. Década de los 90. Concytec. Lima.

CIANIO (2003) : Perú en múmeras 2003. Cuanto. Lima.

GEMAS, Raúl (2002) : Indicadores bibliamétricos de la producción científica penana en el año 2002. Lima.

ESCORSA, Pere (1997) : Manual de Cestióne Innovación Teonológica en la empresa. CINDA. Santiago.

INDECOPI. http://www. indecopi.gob.pe. 2 de octubre de 2004, 18:00 horas.

OAYA GUERRERO, Julio César (2003) : Estudios bibliotecológicas y otros ensayos. Ediciones Generación Conquistadora. Lima.

OAAYA GUERRERO, Julio César et al (2004) : Calidad de los servicios en unidades de información: Gestión y competitividad de bibliotecas. Ediciones Generación Conquistadora. Lima.

OMPI. http://www.wipo.int/about-ip/es/world_ip/2003/dg_message.htm. 18 de febrero de 2004, 20:00 horas. 